TRANSACTIONS OF THE

AMERICAN MATHEMATICAL SOCIETY

Volume 361, Number 2, February 2009, Pages 697-713

S 0002-9947(08)04453-X

Article electronically published on September 26, 2008

\title{
POSITIVE SUPER-SOLUTIONS TO SEMI-LINEAR SECOND-ORDER NON-DIVERGENCE TYPE ELLIPTIC EQUATIONS IN EXTERIOR DOMAINS
}

\author{
VLADIMIR KONDRATIEV, VITALI LISKEVICH, AND ZEEV SOBOL
}

\begin{abstract}
We study the problem of the existence and non-existence of positive super-solutions to a semi-linear second-order non-divergence type elliptic equation $\sum_{i, j=1}^{N} a_{i j}(x) \frac{\partial^{2} u}{\partial x_{i} \partial x_{j}}+u^{p}=0,-\infty<p<\infty$, with measurable coefficients in exterior domains of $\mathbb{R}^{N}$. We prove that in a "generic" situation there is one critical value of $p$ that separates the existence region from nonexistence. We reveal the quantity responsible for the qualitative picture and for the numerical value of the critical exponent which becomes available under a mild stabilization condition at infinity.
\end{abstract}

\section{INTRODUCTION}

We study the existence and non-existence of positive super-solutions to a semilinear second-order non-divergence type elliptic equation

$$
-a \cdot \partial^{2} u=u^{p} \quad \text { in } G
$$

for the full range of the parameter $p \in(-\infty,+\infty)$. Here $G \subset \mathbb{R}^{N}(N \geq 2)$ is an unbounded domain (i.e., connected open set) and $-a \cdot \partial^{2} u:=-\sum_{i, j=1}^{N} a_{i j}(x) \frac{\partial^{2} u}{\partial x_{i} \partial x_{j}}$ is a second-order non-divergence type elliptic expression. We assume throughout the paper that the matrix $a=\left(a_{i j}(x)\right)_{i, j=1}^{N}$ is symmetric measurable and uniformly elliptic, i.e., there exists an ellipticity constant $\nu>0$ such that

$$
\nu^{-1}|\xi|^{2} \leq \sum_{i, j=1}^{N} a_{i j}(x) \xi_{i} \xi_{j} \leq \nu|\xi|^{2}, \quad \text { for all } \xi \in \mathbb{R}^{N} \text { and almost all } x \in G .
$$

The qualitative theory of semi-linear equations of type (1.1) in unbounded domains of different geometries has been extensively studied because of its rich mathematical structure and various applications. One of the features of equation (1.1) in unbounded domains is the non-existence of positive solutions for certain values of

Received by the editors December 20, 2004 and, in revised form, September 15, 2006.

2000 Mathematics Subject Classification. Primary 35J60, 35B33; Secondary 35B05.

Key words and phrases. Non-divergence semi-linear equation, critical exponents.

The research of the first named author was supported by the Institute of Advanced Studies of the University of Bristol via the Benjamin Meaker Fellowship. The second named author was supported by the Forchheimer Visiting Fellowship, Jerusalem. This research was supported in part by the Volkswagen-Stiftung through the RiP-programme at the Mathematisches Forschungsinstitut Oberwolfach, Germany.

(C)2008 American Mathematical Society Reverts to public domain 28 years from publication 
the exponent $p$. Such non-existence phenomena have been known at least since the celebrated paper by Gidas and Spruck [9], where it was proved that the equation

$$
-\Delta u=u^{p}
$$

has no positive classical solutions in $\mathbb{R}^{N}(N \geq 3)$ for $1 \leq p<\frac{N+2}{N-2}$. Though this results is sharp (for $p \geq \frac{N+2}{N-2}$ there are classical positive solutions), the critical exponent $p^{*}=\frac{N+2}{N-2}$ is highly unstable with respect to any changes in the statement of the problem. If one looks for super-solutions to (1.3) in $\mathbb{R}^{N}$ or studies (1.3) in exterior domains, then the value and the properties of the critical exponent change. The following result is well-known: If $N \geq 3$ and $1<p \leq \frac{N}{N-2}$, then there are no positive super-solutions to (1.3) outside a ball in $\mathbb{R}^{N}$. The value of the critical exponent $p^{*}=\frac{N}{N-2}$ is sharp in the sense that (1.3) has (infinitely many) positive solutions outside a ball for any $p>p^{*}$. This statement has been extended in different directions by many authors (see, e.g. [3, 4, 7, 8, 21, 23, 24, and the references therein). In particular, in [13] it was shown that the critical exponent $p^{*}=\frac{N}{N-2}$ is stable with respect to the change of the Laplacian by a second-order uniformly elliptic divergence type operator with measurable coefficients, perturbed by a potential, for a sufficiently wide class of potentials (see also 14 for equations of type (1.3) in exterior domains in the presence of first order terms). In [15, 16] equation (1.3) and the corresponding equation with the divergence type elliptic operators was studied on cone-like domains, and it was shown that the value of the critical exponent is dependent on the geometry of the domain even in the case of the Laplacian, and on the behaviour of the coefficients of the elliptic operator at infinity. The method developed in these papers shows that the exact value of the critical exponent can be determined based upon the precise asymptotics at infinity of the minimal harmonic function with respect to the operator (Green's function) and the "maximal" harmonic function.

In this paper we study the non-divergence type equation (1.1) without any smoothness assumptions on the coefficients. In this generality it is known that the Dirichlet problem is not well posed [19, and there is no potential theory. This makes strong restrictions on the available techniques. Since in the case of smooth coefficients the asymptotic behaviour at infinity of the Green's function of the operator $a \cdot \partial^{2}$ is relatively well understood [1, 20, one can expect that the value of the critical exponent of (1.1) will depend on the behaviour of the coefficients at infinity. Indeed, we show that it is the case, and in a "generic" situation equation (1.1) has one critical exponent which, unlike the case of the divergence type equations, can even move to the sub-linear region $(p<1)$ if the ratio of the maximal and minimal eigenvalues of the matrix $a$ is large enough.

We study strong solutions at infinity to (1.1). More precisely, we say that $u$ is a solution (super-solution, sub-solution) to equation (1.1) at infinity if there exists a closed ball $\bar{B}_{\rho}$ centered at the origin such that if $u \in W_{l o c}^{2, N}\left(\bar{B}_{\rho}^{c}\right), \bar{B}_{\rho}^{c}=\mathbb{R}^{N} \backslash \bar{B}_{\rho}$ and $-a \cdot \partial^{2} u=(\geq, \leq) u^{p}$ a.e. on $\mathbb{R}^{N} \backslash \bar{B}_{\rho}$.

We define the critical exponents to equation (1.1) by

$$
\begin{aligned}
& p^{*}=\inf \{p>1: \text { (1.1) has a positive super-solution at infinity }\}, \\
& p_{*}=\sup \{p<1: \text { (1.1) has a positive super-solution at infinity }\} .
\end{aligned}
$$

The next proposition, whose proof is straightforward, shows that the above values are well defined. 
Proposition 1.1. Let $u>0$ be a solution to the inequality

$$
a \cdot \partial^{2} u+u^{p} \leq 0 \text { on } \bar{B}_{R}^{c}
$$

Then for $q>p>1$ or $q<p<1$ the function $v=u^{\frac{p-1}{q-1}}$ is a positive solution to

$$
a \cdot \partial^{2} v+v^{q} \leq 0 \text { on } \bar{B}_{R}^{c} .
$$

One can expect that the numerical value of the critical exponent will become available if the matrix of coefficients $a=\left(a_{i j}(x)\right)_{i, j=1}^{N}$ tends to a constant matrix $a^{0}=\left(a_{i j}^{0}\right)_{i, j=1}^{N}$ as $|x|$ tends to infinity, and the predicted value of the critical exponent is the same as in the case of the Laplacian. This is indeed true as we will show below, but we reveal much more. It turns out that the quantity responsible for the qualitative picture as well as for the numerical value of the critical exponent is the function

$$
\Psi_{a}(x):=\frac{\operatorname{Tr}(a)}{\frac{(a x, x)}{|x|^{2}}}
$$

which was introduced in [17] where it was called the "effective dimension" for the equation $a \cdot \partial^{2} u=0$. It is the stabilization at infinity of this function that gives the exact numerical value of the critical exponent to (1.1), and the rate of its stabilization determines whether equation (1.1) with the critical value of $p$ has positive super-solutions at infinity. The standard condition on the rate of convergence of the variable coefficients to the constant coefficients in the theorems on proximity of the Green's functions corresponding to the linear equation $a \cdot \partial^{2} u=0$ is the Dini condition at infinity (see [1, 20]). We prove the absence of positive solutions in the critical case under a much more general condition, (1.9), the sharpness of which is shown by a counterexample.

First, observe that $\Psi_{a}$ is invariant under orthogonal transformations but not invariant under affine transformations. Note also that

$$
1<1+(N-1) \nu^{-2} \leq \Psi_{a}(x) \leq 1+(N-1) \nu^{2} .
$$

Let $g$ be a non-degenerate matrix $\operatorname{det} g \neq 0$. Making the change of variables $y=g x$ in (1.1) one obtains

$$
-\sum_{i, j=1}^{N}\left(a_{g}(y)\right)_{i j} \frac{\partial^{2} v}{\partial y_{i} \partial y_{j}}=v^{p}, \text { with } a_{g}(y)=g a\left(g^{-1} y\right) g^{*}, v(y)=u\left(g^{-1} y\right) .
$$

It is clear that the fact of the existence of positive super-solutions to (1.1) does not depend on the change of variables, and the critical exponents $p_{*}$ and $p^{*}$ are the same for (1.1) and for (1.7). In order to formulate the main result we introduce the following quantities:

$$
\begin{aligned}
& \overline{\Psi_{a}}:=\inf _{g} \limsup _{|x| \rightarrow \infty} \Psi_{a_{g}}(x), \\
& \underline{\Psi_{a}}:=\sup _{g} \liminf _{|x| \rightarrow \infty} \Psi_{a_{g}}(x) .
\end{aligned}
$$

Remark 1.2. There exists $g \in G L_{N}$ such that $\overline{\Psi_{a}}=\limsup _{|x| \rightarrow \infty} \Psi_{a_{g}}(x)$. To see this, note that, for $\lambda>0$, the limit points of $\Psi_{a_{\lambda g}}(y)$ as $y \rightarrow \infty$ are independent of $\lambda$. Hence it is enough to consider $g$ of unit Hilbert Schmidt norm in the definition of 
$\bar{\Psi}_{a}$ and $\underline{\Psi}_{a}$. Then the estimate $\Psi_{a_{g}}(y) \geq \frac{1}{\nu^{2}\left|g^{*} \frac{y}{y \mid}\right|^{2}}$ shows that $\bar{\Psi}_{a}$ is attained on a sequence of matrices uniformly bounded from below. Since the unit sphere of matrices is compact, it follows that $\bar{\Psi}_{a}$ is attained on some $g \in G L_{N}$.

In fact, the arguments in the proof of our main theorem (see Section 3) show that for any $g_{1}, g_{2} \in G L_{N}$ one has that $\liminf _{|x| \rightarrow \infty} \Psi_{a_{g_{1}}}(x) \leq \limsup _{|x| \rightarrow \infty} \Psi_{a_{g_{2}}}(x)$, which implies that $\underline{\Psi_{a}} \leq \overline{\Psi_{a}}$.

Now we are ready to formulate the main result of the paper.

Theorem 1.3. Let $\Psi_{a}, \overline{\Psi_{a}}$ be defined in (1.8). Then the following assertions hold:

(i) If $\underline{\Psi}_{a} \geq 2$, then $\bar{p}_{*}=-\infty$ and $1+\frac{2}{\Psi_{a}-2} \leq p^{*} \leq 1+\frac{2}{\underline{\Psi_{a}}-2}$.

(ii) If $\overline{\Psi_{a}} \leq 2$, then $p^{*}=\infty$ and $1-\frac{2}{2-\overline{\Psi_{a}}} \leq p_{*} \leq 1-\frac{2}{2-\underline{\Psi_{a}}}$.

(iii) If $\overline{\Psi_{a}}=\underline{\Psi_{a}}=A \neq 2$ and there exists $g \in G L_{N}$ such that the function

$$
\delta(r):=\sup _{|x| \geq r}\left|\Psi_{a_{g}}(x)-A\right| \rightarrow 0(r \rightarrow \infty)
$$

and

$$
\int^{\infty} \exp \left\{-\frac{2}{|A-2|} \int_{r_{0}}^{r} \delta(s) \frac{d s}{s}\right\} \frac{d r}{r}=\infty \text { for some } r_{0}>0
$$

then (1.1) has no positive super-solutions at infinity for the critical value $p=1+$ $\frac{2}{A-2}$.

(iv) If $\overline{\Psi_{a}}=\underline{\Psi_{a}}=2$, then $p_{*}=-\infty$ and $p^{*}=\infty$, so that (1.1) has no positive super-solutions at infinity for any $p \in \mathbb{R}$.

\begin{tabular}{llll}
\hline \multicolumn{2}{c}{ Non-existence } & Existence \\
\hline-1 & 1 & $p^{*}$ & $p$
\end{tabular}

(i) Case $\liminf _{|x| \rightarrow \infty} \Psi_{a}(x)>2$.

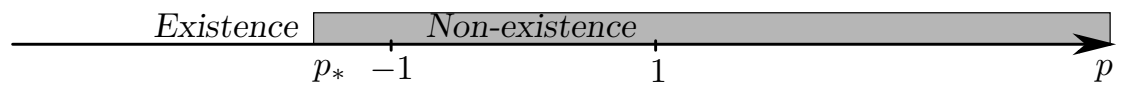

(ii) Case $\lim \sup \Psi_{a}(x)<2$.

$$
|x| \rightarrow \infty
$$

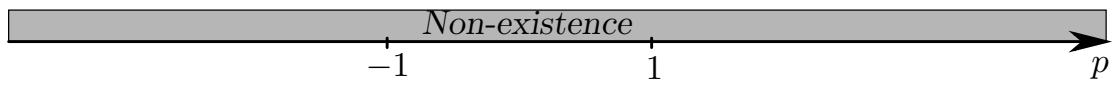

(iv) Case $\limsup _{|x| \rightarrow \infty} \Psi_{a}(x)=\liminf _{|x| \rightarrow \infty} \Psi_{a}(x)=2$.

FiguRE 1. Existence and non-existence regions for equation (1.1).

Some remarks are in order. First, recall that $\varphi:[c, \infty) \rightarrow \mathbb{R}$ is called a Dini function at infinity if

$$
\int_{c}^{\infty} \frac{\varphi(r)}{r} d r<\infty
$$


Remarks 1.4. (a) It is interesting to note that equation (1.1) has no positive supersolutions for all $p \in[-1,1]$, due to (1.6).

(b) Theorem 1.3 describes three distinct cases in existence of positive supersolutions to (1.1) (see also Figure 1). As the examples in Section 3.3 show for the case $\Psi_{a}<2<\overline{\Psi_{a}}$, each of the three situations is possible, and for $\underline{\Psi}_{a}<2=\overline{\Psi_{a}}$ cases (ii) and (iv) are possible. Similarly, for $\underline{\Psi_{a}}=2<\overline{\Psi_{a}}$ cases (i) and (iv) are possible.

(c) If $\delta$ is a Dini function at infinity, then it satisfies condition (1.9).

(d) The next example shows that the conditions of Theorem 1.3 (iii) are optimal. Let $A \neq 2, \varkappa \in \mathbb{R}$. Set

$$
a_{i j}(x)=\delta_{i j}+\gamma(r)\left[\delta_{i j}-\frac{x_{i} x_{j}}{|x|^{2}}\right], \gamma(r)=\frac{A-N+\frac{\varkappa}{\log r}}{N-1} .
$$

Then

$$
\Psi(x)=N+\gamma(r)(N-1)=A+\frac{\varkappa}{\log r}, \delta(r)=\frac{|\varkappa|}{\log r} \rightarrow 0 \text { as } r \rightarrow \infty .
$$

For $|\varkappa| \leq \frac{|A-2|}{2}$ it follows from Theorem 1.3 (iii) that equation (1.1) has no positive super-solutions for $p=\frac{A}{A-2}$. On the other hand, one can readily verify that there exists $R>1$ such that the function

$$
u(x)=c|x|^{2-A}(\log |x|)^{\frac{2-A}{2}}
$$

satisfies the inequality

$$
a \cdot \partial^{2} u+u^{\frac{A}{A-2}} \leq 0
$$

if, for $A>2, \varkappa>\frac{|A-2|}{2}$ with sufficiently small $c>0$, and for $A<2, \varkappa<-\frac{|A-2|}{2}$ with sufficiently large $c>0$. For $\varkappa$ with the opposite sign the equation has no positive super-solutions, as one can see from Propositions 3.8 and 3.14.

Corollary 1.5. Let there exist $a^{0} \in G L_{N}$ such that

$$
\delta(r):=\max _{1 \leq i, j \leq N} \sup _{|x| \geq r}\left|a_{i j}(x)-a_{i j}^{0}\right| \rightarrow 0 \text { as } r \rightarrow \infty .
$$

Then $p_{*}=-\infty$ and $p^{*}=1+\frac{2}{N-2}$. Moreover, if $N \geq 3$ and $\delta$ satisfies (1.9) (in particular, if $\delta$ is a Dini function at infinity), then (1.1) has no positive supersolutions at infinity for $p=p^{*}$.

The rest of the paper is organized as follows. In Section 2 we provide tools that are used in the proof of the main theorem, which is contained in Section 3.

\section{Auxiliary RESUlts ON SUb- AND SUPER-SOlutions}

The following simple lemma is of frequent use in this paper.

Lemma 2.1. Let $f: \mathbb{R}_{+} \rightarrow \mathbb{R}$ and $u(x)=f(|x|)$. Then

$$
a \cdot \partial^{2} u=\frac{(a x, x)}{|x|^{2}}\left(f^{\prime \prime}(|x|)+\frac{\Psi_{a}(x)-1}{|x|} f^{\prime}(|x|)\right) .
$$

In particular, if $u(x)=c|x|^{\alpha}$ we have

$$
a \cdot \partial^{2} u=c \frac{(a x, x)}{|x|^{2}} \alpha|x|^{\alpha-2}\left(\Psi_{a}(x)-2+\alpha\right) .
$$


The next lemma is the key tool in our proofs of non-existence of positive solutions to non-linear equation (1.1).

Lemma 2.2 (Non-existence Lemma). Let $0 \leq V \in L_{l o c}^{\infty}\left(\bar{B}_{R}^{c}\right)$ satisfy

$$
|x|^{2} V(x) \rightarrow \infty \quad \text { as }|x| \rightarrow \infty \text {. }
$$

Then the equation

$$
-a \cdot \partial^{2} u-V u=0 \quad \text { in } \bar{B}_{R}^{c}
$$

has no non-trivial non-negative super-solutions.

The proof consists of a simple re-scaling argument and the use of the following known result [5, Lemma 1.1].

Lemma 2.3. Let $B \subset \mathbb{R}^{N}$ be a unit ball. Then there exists $\lambda>0$ depending on $N$ and $\nu$ only, such that for $\mu>\lambda$ the equation $-a \cdot \partial^{2} u=\mu u$ in $B$ has no positive super-solutions.

In the next two propositions we explore some properties of sub- and supersolutions to the linear equation

$$
a \cdot \partial^{2} u=0
$$

in the exterior domains.

Proposition 2.4. Let $R_{0}>0$. Let $v$ be a sub-solution to (2.4) in $\bar{B}_{R_{0}}^{c}$ such that $\left.v\right|_{\partial B_{R_{0}}} \leq 0$ and $\limsup _{|x| \rightarrow \infty} v(x)>0$. Let $u>0$ be a super-solution to (2.4). Then

$$
\limsup _{|x| \rightarrow \infty} \frac{v(x)}{u(x)}>0 .
$$

Proof. For a contradiction assume that there exists a sequence $\left(R_{n}\right), R_{n} \rightarrow \infty$, such that for any $\varepsilon>0$ there exists $n$ such that $v(x) \leq \varepsilon u(x)$ for any $x \in \bar{B}_{R_{n}}^{c}$. Then by the maximum principle we conclude that $v(x) \leq \varepsilon u(x)$ for any $x \in \bar{B}_{R_{0}}^{c}$ which implies that $v=0$.

Proposition 2.5. Either for all positive super-solutions to (2.4) there holds

$$
\liminf _{|x| \rightarrow \infty} u(x)>0 \text {, }
$$

or for all positive super-solutions to (2.4) there holds

$$
\liminf _{|x| \rightarrow \infty} u(x)<\infty
$$

Proof. Suppose that $u>0$ is a super-solution to (2.4) such that $\liminf _{|x| \rightarrow \infty} u(x)=0$. We can assume without loss that $\left.u\right|_{|x|=R_{0}} \geq 1$. Then $v=1-u$ is a sub-solution to (2.4) satisfying the conditions of Proposition 2.4. Let $w>0$ be a super-solution to (2.4). Since $\lim \sup v(x)=1$, by Proposition 2.4 it follows that

$$
\liminf _{|x| \rightarrow \infty} w(x)<\infty .
$$

Now suppose that $u>0$ is a super-solution to (2.4) such that $\liminf _{|x| \rightarrow \infty} u(x)=\infty$. Without loss assume that $\left.u\right|_{|x|=R_{0}}>1$. Then $w_{\varepsilon}:=u^{-\varepsilon}-\varepsilon$ is a sub-solution 
decaying to $-\varepsilon$. By the maximum principle for any super-solution $w>0$ there exists a constant $c$ such that

$$
w \geq c\left(u^{-\varepsilon}-\varepsilon\right), \quad c=\inf _{|x|=1} w .
$$

Therefore for any fixed $R$ denoting $M_{R}=\max _{R_{0} \leq|x| \leq R} u(x)$ we have that

$$
(|x|<R) \Longrightarrow w(x) \geq c\left(M_{R}^{-\varepsilon}-\varepsilon\right) \rightarrow c \text { as } \varepsilon \rightarrow 0,
$$

which implies that $\liminf _{|x| \rightarrow \infty} u>0$.

The following proposition provides an a priori estimate for super-solutions to (1.1) in the sub-linear case.

Proposition 2.6. Let $p<1$. Let $R_{0}>0$. Let $u$ be a positive solution to

$$
a \cdot \partial^{2} u+u^{p} \leq 0 \text { in } \bar{B}_{R_{0}}^{c} .
$$

Then there exists $c>0$ such that

$$
u(x) \geq c|x|^{\frac{2}{1-p}} .
$$

The proof of the above proposition is based upon the next lemma (cf. [12]), where we use the following notation $A_{r, R}:=\left\{x \in \mathbb{R}^{N}: r<|x|<R\right\}$.

Lemma 2.7. Let $q>1$. Let $v$ be a positive solution to

$$
a \cdot \partial^{2} v \geq v^{q} \text { in } A_{1 / 2,5 / 2} .
$$

Then there is a constant $c=c(\lambda, N, q)$ such that

$$
v(x) \leq c \text { for all } x \in A_{1,2} \text {. }
$$

Proof. We choose a constant $\varkappa$ such that

$$
w(x)=\varkappa\left(25 / 4-|x|^{2}\right)^{2 /(1-q)}\left(|x|^{2}-1 / 4\right)^{2 /(1-q)}
$$

is a solution to

$$
a \cdot \partial^{2} w \leq w^{q} \text { in } A_{1 / 2,5 / 2} .
$$

Since $w=\infty$ on $\partial A_{1 / 2,5 / 2}$ by the maximum principle we conclude that

$$
v \leq w .
$$

In particular, $\max _{A_{1,2}} v(x) \leq \max _{A_{1,2}} w(x)$.

Proof of Proposition 2.6. Set $v=\frac{1}{u}, q=2-p$. Then $v$ satisfies the inequality

$$
a \cdot \partial^{2} v \geq v^{q} \text { in } \bar{B}_{R_{0}^{c}}
$$

with $q>1$. Let $R>>R_{0}$. Scaling the annulus $A_{1 / 2 R, 5 / 2 R}$ by changing variables as follows,

we obtain that $\tilde{v}$ solves

$$
y=\frac{x}{R} \quad \text { and } \quad \tilde{v}(y)=R^{\frac{2}{q-1}} v(x),
$$

$$
a \cdot \partial^{2} \tilde{v} \geq \tilde{v}^{q} \text { in } A_{1 / 2,5 / 2} .
$$

By Lemma 2.7 we have that

$$
\max _{A_{R, 2 R}} v(x) \leq c R^{\frac{2}{1-q}},
$$

which implies the assertion. 
Lemma 2.8. Let $f:(0, \infty) \rightarrow(0, \infty)$ be such that for any $c>0, \inf _{t \in(c, \infty)} \frac{f(t)}{t}>0$.

Let $u$ be a non-negative solution to

$$
a \cdot \partial^{2} u+f(u) \leq 0 \text { in } \bar{B}_{R_{0}^{c}} .
$$

Then $\liminf _{|x| \rightarrow \infty} u(x)=0$.

Proof. For a contradiction, suppose that $\liminf _{|x| \rightarrow \infty} u(x)>0$. Then there exist $c>0$ and $R>R_{0}$ such that $u(x) \geq c$ on $\bar{B}_{R}^{c}$. Let $\inf _{t \in(c, \infty)} \frac{f(t)}{t}=m$. Therefore

$$
a \cdot \partial^{2} u+m u \leq 0
$$

which contradicts Lemma 2.2

Corollary 2.9. For equation (1.1) there exists at most one (finite) critical exponent.

Proof. For a contradiction, assume that there are $p_{1}<1<p_{2}$ and $u_{1}, u_{2}$ positive such that $a \cdot \partial^{2} u_{j}+u_{j}^{p_{j}} \leq 0, j=1,2$. Proposition 2.6 implies that

$$
\lim _{x \rightarrow \infty} u_{1}(x)=\infty
$$

By Lemma 2.8 we have that

$$
\liminf _{|x| \rightarrow \infty} u_{2}(x)=0 .
$$

This contradicts Proposition 2.5.

\section{Proof of the main theorem}

3.1. Case $p \geq 1$.

Proposition 3.1. Let $g \in G L_{N}$ be such that

$$
n(g):=\liminf _{|x| \rightarrow \infty} \Psi_{a_{g}}(x)>2 .
$$

Let $p>1+\frac{2}{n(g)-2}$. Then there exist $R>0$ and $c>0$ such that $u(x)=c|g x|^{-\frac{2}{p-1}}$ is a super-solution to (1.1) in $\bar{B}_{R}^{c}$.

Proof. The assertion follows from (2.1). Note that the choice $\alpha=-\frac{2}{p-1}$ provides $\alpha-2=\alpha p$ and $\alpha\left(\Psi_{a_{g}}(x)-2+\alpha\right)<0$ since $\alpha<0$ and $\Psi_{a_{g}}(x)-2+\alpha>$ $n(g)-2-\frac{2}{p-1}>0$.

Proposition 3.2. Let $g \in G L_{N}$ be such that

$$
N(g):=\limsup _{|x| \rightarrow \infty} \Psi_{a_{g}}(x) \geq 2 .
$$

Let $1 \leq p<1+\frac{2}{N(g)-2}$. Then equation (1.1) has no positive super-solutions at infinity.

Remark 3.3. For $N(g)=2$ the above means that there are no positive supersolutions to (1.1) for all $p \geq 1$. 
Proof. For a contradiction suppose that there exists $R_{0}>0$ and $u>0$, a supersolution to (1.1) in $\bar{B}_{R_{0}}^{c}$ Choose $\beta$ such that $-\frac{2}{p-1}<\beta<2-N(g)$ (for $p=1$ chose $\beta<2-N(g))$. Then there exists $R>R_{0}$ such that $\Psi_{a_{g}}(x)-2+\beta<0$ on $B_{R}^{c}$. Hence by (2.1) $v(x)=|g x|^{\beta}$ is a sub-solution to (2.4). Then by the maximum principle there exists a constant $c>0$ such that

$$
u(x) \geq c|g x|^{\beta} \text { on } \bar{B}_{R}^{c} .
$$

Since $\beta(p-1)>-2$, there exists $\gamma>0$ such that $u$ satisfies the inequality

$$
a \cdot \partial^{2} u+c|g x|^{-2+\gamma} u \leq 0 \text { on } \bar{B}_{R}^{c}
$$

which contradicts Lemma 2.3

\subsection{Case $p<1$.}

Proposition 3.4. Let $g \in G L_{N}$ be such that $N(g)<2$. Let $p<1+\frac{2}{N(g)-2}$. Then there exist $R>0$ and $c>0$ such that $u(x)=c|g x|^{\frac{2}{1-p}}$ is a super-solution to (1.1) in $\bar{B}_{R}^{c}$.

Proof. Direct computation using Lemma 2.1.

Proposition 3.5. Let $g \in G L_{N}$ be such that $n(g) \leq 2$. Let $1+\frac{2}{n(g)-2}<p<1$. Then equation (1.1) has no positive super-solutions at infinity.

Remark 3.6. For $n(g)=2$ the above means that there are no positive supersolutions to (1.1) for all $p<1$.

Proof of Proposition 3.5. Choose $\alpha>0$ such that $\frac{2}{1-p}>\alpha>2-n(g)$. It is straightforward that the function $v(x)=|g x|^{\alpha}$ is a growing sub-solution to (2.4) at infinity. By Proposition 2.6, a non-zero super-solution $u$ to (1.1) satisfies estimate (2.5) So that $\frac{v(x)}{u(x)} \rightarrow 0$ as $|x| \rightarrow \infty$, in contradiction to Proposition 2.4.

Remark 3.7. Now we are ready to justify the inequality $\underline{\Psi_{a}} \leq \overline{\Psi_{a}}$. Assume for a contradiction that there exist $g_{1}, g_{2} \in G L_{N}$ such that $n\left(g_{1}\right)>N\left(g_{2}\right)$. If $2 \leq N\left(a_{g_{2}}\right)<n\left(a_{g_{1}}\right)$, then by Proposition 3.1 equation (1.1) has no positive supersolutions for $1<p<1+\frac{2}{N\left(a_{g_{2}}\right)-2}$, and by Proposition 3.1 positive super-solutions exist for $p>1+\frac{2}{n\left(a_{g_{1}}\right)-2}$. Contradiction. For $N\left(a_{g_{2}}\right)<n\left(a_{g_{1}}\right) \leq 2$, a contradiction is obtained from Propositions 3.4 and 3.5. If $N\left(a_{g_{2}}\right)<2<n\left(a_{g_{1}}\right)$, Propositions 3.1 and 3.4 imply that (1.1) has two finite critical exponents, which contradicts Corollary 2.9.

The assertions (i), (ii) and (iv) of Theorem 1.3 follow directly from Propositions 3.1 3.5. Corollary 2.9 and definition (1.8).

3.3. Case $\Psi_{a} \leq 2 \leq \overline{\Psi_{a}}$. Examples. In this section we provide examples showing that all the qualitative situations described in Theorem 1.3 (i),(ii),(iv) may occur in this case. We study the inequality

$$
\sum_{i, j=1}^{N} a_{i j} \frac{\partial^{2} u}{\partial x_{i} \partial x_{j}}+u^{p} \leq 0
$$

with the matrix (see, e.g. 17])

$$
a_{i j}(x)=\delta_{i j}+\gamma(r) \frac{x_{i} x_{j}}{r^{2}}, r=|x| .
$$


For the respective matrix $a_{g}$ (recall (1.7)) we obtain

$$
\Psi_{a_{g}}(y)=1+\frac{\operatorname{Tr}\left(g g^{*}\right)-\frac{\left|g^{*} y\right|^{2}}{|y|^{2}}}{\frac{\left|g^{*} y\right|^{2}}{|y|^{2}}+\gamma\left(\left|g^{-1} y\right|\right) \frac{|y|^{2}}{\left|g^{-1} y\right|^{2}}} .
$$

Taking $y$ along the directions of eigenvectors of $g g^{*}$ one can see the validity of the inequality

$$
\underline{\Psi_{a}}=1+\frac{N-1}{1+\limsup _{r \rightarrow \infty} \gamma(r)} \leq 1+\frac{N-1}{1+\liminf _{r \rightarrow \infty} \gamma(r)}=\overline{\Psi_{a}} .
$$

Therefore the case under discussion occurs if

$$
\liminf _{r \rightarrow \infty} \gamma(r) \leq N-2 \leq \limsup _{r \rightarrow \infty} \gamma(r) .
$$

Let $\varepsilon=\frac{2-N+\gamma}{N-1}$. Then $\liminf _{r \rightarrow \infty} \varepsilon \leq 0 \leq \limsup _{r \rightarrow \infty} \varepsilon$. For $u$ spherically symmetric inequality (3.1) takes the form

$$
\frac{\partial^{2} u}{\partial r^{2}}+\frac{1}{r(1+\varepsilon)} \frac{\partial u}{\partial r}+\frac{1}{1+\varepsilon} \frac{1}{N-1} u^{p} \leq 0 .
$$

We assume further that the function $\varepsilon\left(\mathrm{e}^{t}\right)$ is periodic with period $T$ and such that $\liminf \varepsilon>-1$. Then the factor $\frac{1}{(1+\varepsilon)(N-1)}$ makes no difference, so we omit it further on. Let

$$
\varkappa=\frac{1}{T} \int_{0}^{T} \frac{d \tau}{1+\varepsilon\left(\mathrm{e}^{\tau}\right)}
$$

We distinguish three cases: $\varkappa>1, \varkappa<1$ and $\varkappa=1$.

$\varkappa>1$. Following [2] (see also [22]) we use the transformation

$$
s=\left(\int_{r}^{\infty} \frac{d r}{\rho(r)}\right)^{-1}, y(s)=s u(r), \text { where } \frac{\rho^{\prime}}{\rho}=\frac{1}{r(1+\varepsilon)}, \rho(r) \asymp r^{\varkappa} .
$$

Then we obtain in new variables

$$
y^{\prime \prime}+s^{-p-3} \rho^{2}(r(s)) y^{p} \leq 0 .
$$

The latter has a positive solution on $(1, \infty)$ if $\int_{1}^{\infty} s^{-p-2} \rho^{2}(r(s)) d s<\infty$, which is fulfilled if $p>1+\frac{2}{\varkappa-1}$. At the same time, if $\varepsilon>-1+\beta$ with some $\beta \in(0,1)$, then for $\alpha<1-\frac{1}{\beta}$ one can directly verify that $r^{\alpha}$ is a sub-solution to the linear equation corresponding to (3.1). By the maximum principle, a non-zero super-solution $u$ of (2.4) satisfies $u \geq c r^{\alpha}$. Hence a non-zero super-solution of (1.1) with $p \geq 1$ should satisfy at infinity the inequality $a \cdot \partial^{2} u+c r^{\alpha(p-1)} u \leq 0$. For $p<1+\frac{2}{|\alpha|}$ it contradicts Lemma 2.2,

Therefore we conclude that for this case there exists a non-trivial critical exponent $p^{*}>1$. This realizes the qualitative case (i) (see Figure 1).

$\varkappa<1$. Using the same notation as above we introduce a new independent variable by $d t=\frac{d r}{\rho(r)}$. Then we obtain the inequality

$$
u_{t t}+\rho^{2}(r(t)) u^{p} \leq 0 .
$$

One can directly verify that there exists $c>0$ such that $u(t)=c t^{\alpha}$ solves the above inequality if $\frac{2}{(1-\varkappa)(1-p)} \leq \alpha<1$. Therefore if $p<1-\frac{2}{1-\varkappa}$ inequality (3.1) has a positive solution. On the other hand, $u(t)=t$ solves the linear equation $u_{t t}=0$. Note that $t \asymp r^{1-\varkappa}$. So if (3.1) has a positive solution $u$ by Proposition 2.4, we 
have that $\liminf _{|x| \rightarrow \infty} \frac{u}{|x|^{1-\varkappa}}<\infty$. At the same time, from Proposition 2.6 it follows that $u \geq c r^{\frac{2}{1-p}}$. Hence $p \leq 1-\frac{2}{1-\varkappa}$. So this realizes case (ii).

$\varkappa=1$. In this case the linear part has a solution $\asymp \log r$. It is easy to see that this implies absence of positive solutions to (3.1) for all $-\infty<p<\infty$.

It is easy to produce $\varepsilon$ as above such that $\liminf _{r \rightarrow \infty} \varepsilon<0<\limsup _{r \rightarrow \infty} \varepsilon$, which realizes each of the three cases: $\varkappa>1, \varkappa=1$ and $\varkappa<1$. One can also produce $\varepsilon$ satisfying $\liminf _{r \rightarrow \infty} \varepsilon<0=\limsup _{r \rightarrow \infty} \varepsilon$ and realizing the cases $\varkappa>1$ and $\varkappa=1$, or satisfying $\liminf _{r \rightarrow \infty} \varepsilon=0<\limsup _{r \rightarrow \infty}^{r \rightarrow \infty} \varepsilon$ and realizing the cases $\varkappa<1$ and $\varkappa=1$.

3.4. Stabilization: $\underline{\Psi}_{a}=\overline{\Psi_{a}}=A>2$. In this subsection we prove assertion (iii) of Theorem 1.3 for the case $A>2$. The required result follows from the next proposition.

Proposition 3.8. Let $A>2, \delta(r) \downarrow 0$ as $r \rightarrow \infty$. Let $\Psi_{a}$ defined by (1.5) satisfy the following inequality:

$$
2 \leq \Psi_{a}(x) \leq A+\delta(|x|),|x|>r_{0},
$$

for some $r_{0}>1$ with $\delta$ such that

$$
\int^{\infty} \exp \left\{-\frac{2}{A-2} \int_{r_{0}}^{r} \delta(s) \frac{d s}{s}\right\} \frac{d r}{r}=\infty .
$$

Then there is no positive super-solution at infinity to equation (1.1) with $p=1+$ $\frac{2}{A-2}$.

Throughout the subsection we assume the conditions of Proposition 3.8 to be fulfilled. We split the proof of Proposition 3.8 into several lemmas, with the following notation:

$$
\Lambda(r):=\exp \left\{\int_{r_{0}}^{r} \delta(s) \frac{d s}{s}\right\}
$$

Lemma 3.9. Let $u$ be a positive super-solution to (1.1). Then there is a constant $c>0$ such that

$$
\frac{u(r)}{c} \geq v_{0}(r):=\int_{r}^{\infty} \frac{d s}{s^{A-1} \Lambda(s)} .
$$

Proof. Note that $v_{0}$ is a well-defined function vanishing at infinity, since $A>2$ and $\delta(r) \downarrow 0$ as $r \rightarrow \infty$. By (3.4), $v_{0}$ is a sub-solution of the equation $a \cdot \partial^{2} v=0$ (vanishing at infinity). Since $u$ is a positive super-solution of the same equation, the assertion follows.

Further on, assuming the existence of a positive super-solution $u$ to (1.1), we study the following equation on $\left(r_{0}, \infty\right)$ :

$$
v_{r r}+\frac{1}{r} v_{r}^{+}-\frac{A-1+\delta}{r} v_{r}^{-}+\frac{1}{M} \tilde{u}^{\frac{2}{A-2}} v=0,
$$

with $\tilde{u}(r):=\min _{|x|=r} u(x)$ and $M>0$. From Lemma 2.1 and assumption (3.4), the existence of a positive solution $v$ to (3.7) with $M$ large enough, implies that there exists a positive sub-solution $w$ to the equation $a \cdot \partial^{2} w+u^{p-1} w=0$ (without loss of generality we assume that $M=1), w(x):=v(|x|)$. 
Lemma 3.10. The positive solution $v$ of (3.7) with the initial data $v\left(r_{0}\right)=0$, $v_{r}\left(r_{0}\right)=1$, has a unique critical point $r_{M} \in\left(r_{0}, \infty\right)$, which is the point of the only local maximum. The mapping $M \mapsto r_{M}$ is continuous and $r_{M} \rightarrow \infty$ as $M \rightarrow \infty$.

Proof. If $v$ is increasing on $\left(r_{0}, \infty\right)$, then (3.7) implies $\left(r v_{r}\right)_{r}+c \frac{r}{M} \tilde{u}^{\frac{2}{A-2}} v \leq 0$ with some constant $c>0$. Since $r v_{r}>0$ it follows that $\int_{r_{0}}^{\infty} r \tilde{u}^{\frac{2}{A-2}} v d r<\infty$. On the other hand, Lemma 3.9 together with (3.5) yield

$$
\int_{r_{0}}^{\infty} r \tilde{u}^{\frac{2}{A-2}} v d r \geq v\left(r_{0}+1\right) \int_{r_{0}+1}^{\infty} \Lambda^{-\frac{2}{A-2}} \frac{d r}{r}=\infty
$$

which is a contradiction. Hence there exists $r_{M} \in(0, \infty)$ such that $v_{r}\left(r_{M}\right)=0$. Since $r v_{r}$ decreases, $v_{r}(r)<0$ for $r>r_{M}$.

Since $r \mapsto v_{r}(r)$ is a diffeomorphism, $C^{\infty}$-smooth in $M$, we conclude that the mapping $M \mapsto r_{M}$ is continuous. We have

$$
r_{0}=-\left.r v_{r}(r)\right|_{r=r_{0}} ^{r=r_{M}}=\frac{c}{M} \int_{r_{0}}^{r_{M}} \tilde{u}^{\frac{2}{A-2}}(r) \int_{r_{0}}^{r} s v_{r}(s) \frac{d s}{s} d r \leq \frac{c r_{0}}{M} \int_{r_{0}}^{r_{M}} \tilde{u}^{\frac{2}{A-2}}(r) \ln \left(\frac{r}{r_{0}}\right) d r
$$

which implies that

$$
M \leq c \int_{r_{0}}^{r_{M}} \tilde{u}^{\frac{2}{A-2}}(r) \ln \left(\frac{r}{r_{0}}\right) d r .
$$

Hence $r_{M} \rightarrow \infty$ as $M \rightarrow \infty$.

Lemma 3.11. Let $u$ be a positive super-solution to (1.1) with $p=1+\frac{2}{A-2}$. Then

$$
\int_{r_{1}}^{\infty} \theta^{2} \tilde{u}^{\frac{2}{A-2}} r^{A-1} \Lambda(r) d r \leq \int_{r_{1}}^{\infty} \theta_{r}^{2} r^{A-1} \Lambda(r) d r, \theta \in C_{c}^{1}\left(\mathbb{R}_{+}\right)
$$

Proof. Let $v$ be a positive solution of (3.7) with $M=1$ and the initial data $v\left(r_{0}\right)=$ $0, v_{r}\left(r_{0}\right)=1$ so that $r_{1}$ is the maximum point of $v$. Then either $v(R)=0$ for some $R \in\left(r_{1}, \infty\right)$, or $v$ can be extended to the whole semi-axis. In the former case $w(x):=v(|x|)$ is a positive sub-solution to $a \cdot \partial^{2} w+u^{\frac{2}{A-2}} w=0$ vanishing on both spheres $\left\{|x|=r_{0}\right\}$ and $\{|x|=R\}$. Then, by the maximum principle, $u \geq \alpha w$ for all $\alpha>0$, i.e., $u \equiv+\infty$. This contradicts the existence of $u$. Hence we conclude that $v$ can be extended to a positive solution of (3.7) on the whole semi-axis.

For $r>r_{1}$, (3.7) takes the following form:

$$
-\left(r^{A-1} \Lambda(r) v_{r}\right)_{r}=r^{A-1} \Lambda(r) \tilde{u}^{\frac{2}{A-2}} v .
$$

For $\theta \in C_{c}^{1}\left(\mathbb{R}_{+}\right)$, multiply the latter by $\frac{\theta^{2}}{v}$ and integrate on $\left(r_{1}, \infty\right)$. One obtains the following:

$$
2 \int_{r_{1}}^{\infty} \theta \frac{v_{r}}{v} \theta_{r} r^{A-1} \Lambda(r) d r-\int_{r_{1}}^{\infty} \theta^{2} \frac{v_{r}^{2}}{v^{2}} r^{A-1} \Lambda(r) d r=\int_{r_{1}}^{\infty} \theta^{2} r^{A-1} \Lambda(r) \tilde{u}^{\frac{2}{A-2}} d r .
$$

Now the assertion follows by the Cauchy inequality. 
Lemma 3.12. Let $M>1, r_{M}$ be defined in Lemma 3.10, $\rho>r_{M}, 0 \leq Q(r)<$ $\frac{1}{M} \tilde{u}^{\frac{2}{A-2}}$. Then there exists a unique solution $v$ to the boundary value problem

$$
-\left(r^{A-1} \Lambda(r) v_{r}\right)_{r}=r^{A-1} \Lambda(r) Q(r) v, v\left(r_{M}\right)=\tilde{u}\left(r_{M}\right), v(\rho)=0 .
$$

Moreover, $v$ is a decreasing function, $0<v<\tilde{u}$ and

$$
\int_{r_{M}}^{\rho} v_{r}^{2} r^{A-1} \Lambda(r) d r<c_{M}
$$

with some constant $c_{M}$ dependent on $M$ and $\tilde{u}$ only.

Proof. The existence, uniqueness and positivity of $v$ follow from Lemma 3.11 via the Lax-Milgram theorem. Note that, by the mean value theorem, $v_{r}(s)<0$ for some $s \in\left[r_{M}, \rho\right]$. For a contradiction assume that $v$ is not decreasing. Then it follows from (3.8) that $v_{r r}<0$, and $v$ attains the only maximum at some $r^{\prime} \in\left(r_{M}, \rho\right)$ so that $v_{r}\left(r^{\prime}\right)=0$. Define $\hat{v} \in C^{1}\left(r_{0}, \rho\right)$ in the following way: $\hat{v}$ is the solution to

$$
\left(r \hat{v}_{r}\right)_{r}+\frac{r}{M} \tilde{u}^{\frac{2}{A-2}} \hat{v}=0, \quad \hat{v}\left(r_{0}\right)=0, \quad \hat{v}_{r}\left(r_{0}\right)=1
$$

on $\left(r_{0}, r_{M}\right)$. (Note that $\hat{v}_{r}\left(r_{M}\right)=0$ by the definition of $r_{M}$.) Set $\hat{v}(r)=\hat{v}\left(r_{M}\right)$ for $r \in\left[r_{M}, r^{\prime}\right]$, and $\hat{v}(r):=\frac{\hat{v}\left(r^{\prime}\right)}{v\left(r^{\prime}\right)} v(r)$ for $r \in\left[r^{\prime}, \rho\right]$. Then $\hat{v}$ is a positive sub-solution to the Dirichlet problem for (3.7) on $\left(r_{0}, \rho\right)$ with zero boundary conditions. Hence $w(x):=\hat{v}(|x|)$ is a positive sub-solution to $a \cdot \partial^{2} w+u^{\frac{2}{A-2}} w=0$ vanishing on both spheres $\left\{|x|=r_{0}\right\}$ and $\{|x|=\rho\}$. Then, by the maximum principle (see [5, p. 48]), $u \geq \alpha w$ for all $\alpha>0$, i.e., $u \equiv+\infty$. This contradiction implies that $v$ is decreasing. Therefore, $w(x)=v(|x|), r_{M}<|x|<\rho$, is a sub-solution to the equation $a \cdot \partial^{2} w+u^{\frac{2}{A-2}} w=0$. Hence by the maximum principle $w \leq u$.

Let $\eta \in C^{\infty}\left(r_{M}, r_{M}+1\right), \eta\left(r_{M}\right)=\tilde{u}\left(r_{M}\right)$, and $\eta(s)=0$ for $s>r_{M}+1 / 2$. Now choose $v-\eta$ as a test function for (3.8). The last assertion follows by integration by parts.

Corollary 3.13. For $M>1$, there exists a positive solution to the following equation on $\left(r_{M}, \infty\right)$ :

$$
\left(r^{A-1} \Lambda(r) v_{r}\right)_{r}+\frac{1}{M} r^{A-1} \Lambda(r) v^{1+\frac{2}{A-2}}=0 .
$$

Proof. It follows from Lemmas 3.11 and 3.12 that, for $0 \leq Q(r)<\frac{1}{M} \tilde{u}^{\frac{2}{A-2}}$, there exists a unique solution to the problem on $\left(r_{M}, \infty\right)$ :

$$
\begin{gathered}
\left(r^{A-1} \Lambda(r) v_{r}\right)_{r}+\frac{1}{M} r^{A-1} \Lambda(r) Q(r) v=0, v\left(r_{M}\right)=\tilde{u}\left(r_{M}\right), \\
\int_{r_{M}}^{\infty} r^{A-1} \Lambda(r)\left|v_{r}\right|^{2} d r<\infty,
\end{gathered}
$$

and $0<v \leq \tilde{u}$. Moreover, the maximum principle shows that, in fact, $v \geq c v_{0}(r)$ with $v_{0}$ from Lemma 3.9 and some $c>0$.

Define the set

$$
S:=\left\{w \in C\left[r_{M}, \infty\right]: c v_{0} \leq w \leq \tilde{u}, \int_{r_{M}}^{\infty} r^{A-1} \Lambda(r)\left|w_{r}\right|^{2} d r<c_{M}\right\},
$$

with $c_{M}$ as in Lemma 3.12 Let $F: S \rightarrow C\left[r_{M}, \infty\right]$ be the map $F: w \mapsto v$ such that $v$ is the solution of (3.11) with $Q=\frac{1}{M} w^{\frac{2}{A-2}}$. Then $S$ is a convex compact 
in $C\left[r_{M}, \infty\right], F S \subset S$. It is readily seen that the graph of $F$ is closed (uniqueness of the weak solution to (3.11)). Therefore by the Kakutani theorem (see, e.g. [11, Chap. XVI, §5]) the map $F$ has a fixed point. Hence the assertion follows.

Proof of Proposition 3.8, Let us change the variables in (3.10):

$$
t(r):=\left(\int_{r}^{\infty} \frac{d s}{s^{A-1} \Lambda(s)}\right)^{-1}, w(t):=t v(r(t)) .
$$

Then the equation takes the form

$$
w_{t t}+\frac{1}{M} r^{2 A-2}(t) \Lambda(r(t)) t^{-4-\frac{2}{A-2}} w^{1+\frac{2}{A-2}}=0, t_{M}<t<\infty .
$$

Now by [2], equation (3.12) has no positive solutions if and only if

$$
\int_{t_{M}}^{\infty} r^{2 A-2}(t) \Lambda(r(t)) t^{-3-\frac{2}{A-2}} d t=\infty .
$$

Note that $(A-2) r^{A-2} \Lambda(r) \leq t \leq(A-2+\delta(r)) r^{A-2} \Lambda(r)$. Therefore (3.10) has no positive solutions if and only if (3.5) holds.

3.5. Stabilization: $\Psi_{a}=\overline{\Psi_{a}}=A<2$. Here by proving the following proposition we complete the proof of assertion (iii) in Theorem 1.3 .

Proposition 3.14. Let $1<A<2, \delta(r) \downarrow 0$ as $r \rightarrow \infty$. Let $\Psi_{a}$, defined by (1.5), satisfy the following inequality:

$$
A-\delta(|x|) \leq \Psi_{a}(x) \leq 2,|x|>r_{0},
$$

for some $r_{0}>1$ with $\delta$ such that

$$
\int^{\infty} \exp \left\{-\frac{2}{2-A} \int_{r_{0}}^{r} \delta(s) \frac{d s}{s}\right\} \frac{d r}{r}=\infty .
$$

Then there is no positive super-solution at infinity to equation (1.1) with $p=1+$ $\frac{2}{A-2}$.

Note that $A>1$. Consider the initial value problem

$v_{r r}+\frac{A-1-\delta(r)}{r} v_{r}^{+}-\frac{1}{r} v_{r}^{-}+v^{\frac{A}{A-2}}=0 \quad$ for $r>r_{0} ; \quad v\left(r_{0}\right)=\varepsilon, \quad v_{r}\left(r_{0}\right)=K$.

Let $(\varepsilon, R), R \leq \infty$, be the maximal right interval of existence of the solution $v$ to (3.15) in the region $\left\{(r, v) \in\left(r_{0},+\infty\right) \times(0,+\infty)\right\}$. Let $\Lambda(r)$ be defined by (3.6). Then (3.14) takes the form

$$
\int^{\infty} \Lambda(r)^{-\frac{2}{2-A}} \frac{d r}{r}=\infty
$$

Lemma 3.15. $\quad(i)$ The solution $v$ to (3.15) has exactly one maximum and no minima, and $R<\infty$.

(ii) For any $r_{1}>r_{0}, M>1$ there exists $K>K_{0}$ such that $\max _{\left[r_{0}, r_{1}\right]} v \geq M$.

Proof. Note that on the set $\left\{v_{r}<0\right\}$ the function $v$ satisfies the following equation:

$$
\left(r v_{r}\right)_{r}+r v^{\frac{A}{A-2}}=0 .
$$

Hence if $\left\{v_{r}<0\right\}$ is non-empty, then $\left\{v_{r}<0\right\}=\left(r_{\max }, R\right)$, where $r_{\max }$ is the point of the local maximum which is the only extremum, and $R$ is finite since $v$ is concave 
in $t=\log r$ (13.17) implies that $\left.v_{t t} \leq 0\right)$. Thus, to prove assertion (i) it suffices to show that the set $\left\{v_{r}>0\right\}$ is bounded.

Set $\rho(r):=r^{A-1} \Lambda(r)^{-1}$. Then for $r \in\left\{v_{r}>0\right\}$ the following equation holds:

$$
\left(\rho(r) v_{r}\right)_{r}+\rho(r) v^{\frac{A}{A-2}}=0 .
$$

Changing the independent variable $r=r(t)$,

$$
t=\int_{r_{0}}^{r} \frac{d s}{\rho(s)}, t_{1}=t\left(r_{1}\right)
$$

we see that $\underline{c} r^{2-A} \Lambda(r)<t<\bar{c} r^{2-A} \Lambda(r)$ for some $\bar{c}>\underline{c}>0$. Moreover, $v_{t} \geq 0$, and (3.18) takes the form

$$
v_{t t}+\rho^{2}(r(t)) v^{\frac{A}{A-2}}=0 .
$$

Since $v$ is an increasing concave function of $t$, it follows that $\varepsilon \leq v \leq \varepsilon+K \rho\left(r_{0}\right) t$ and hence

$$
C \varepsilon^{-\frac{A}{2-A}} t^{\frac{2 A-2}{2-A}} \Lambda^{-\frac{2}{2-A}} \geq-v_{t t} \geq c t^{-1} \Lambda^{-\frac{2}{2-A}}(r(t)) .
$$

If $v_{t}$ does not change the sign on an infinite interval, the next integral converges, in contradiction to (3.14):

$$
\infty>\int^{\infty}\left(-v_{t t}\right) d t \geq \int^{\infty} \Lambda^{-\frac{2}{2-A}}(r(t)) \frac{d t}{t} \asymp \int^{\infty} \Lambda^{-\frac{2}{2-A}}(r) \frac{d r}{r} .
$$

Thus, (i) follows.

Now we prove the last assertion. Let $t_{\max }=t\left(r_{\max }\right)$ be the point where $v$ attains its maximum. Then, by (3.21), $K$ and $t_{\max }$ obey

$$
K=-\int_{t_{0}}^{t_{\max }} v_{t t} d t \leq C \varepsilon^{-\frac{A}{2-A}} \int_{t_{0}}^{t_{\max }} t^{\frac{2 A-2}{2-A}} \Lambda^{-\frac{2}{2-A}} d t .
$$

Hence $t_{\max } \rightarrow \infty$ as $K \rightarrow \infty$, in particular $t_{\max }>t_{1}$ for $K$ large enough. By the Landau inequality,

$$
\max _{\left[t_{0}, t_{1}\right]} v_{t}^{2} \leq C \max _{\left[t_{0}, t_{1}\right]} v \max _{\left[t_{0}, t_{1}\right]}\left|v_{t t}\right|
$$

So, by (3.21),

$$
\max _{\left[t_{0}, t_{1}\right]} v \geq c \varepsilon^{\frac{A}{2-A}} K^{2}
$$

Proof of Proposition 3.14. For a contradiction, assume that $u>0$ is a supersolution to (1.1) with $p=\frac{A}{A-2}$. Choose $c>0$ such that $\frac{(a x, x)}{|x|^{2}} c^{1-p} \leq 1$. Let $\varepsilon>0$ be such that $\varepsilon c \leq \min _{|x|=r_{0}} u(x)$. For $K>0$, let $v$ be the solution to the problem (3.15). Then $w(x)=c v(|x|)$ is a solution to $a \cdot \partial^{2} w+w^{p} \geq 0$ such that $u(x) \geq w(x)$ on $\partial B_{r_{0}}$. Since $p<0$ it follows from the maximum principle that $u \geq w$ on $\bar{B}_{r_{0}}^{c}$. On the other hand, by Lemma $3.15 w$ reaches arbitrary large values on the annulus $r_{0} \leq|x| \leq 2 r_{0}$. Contradiction. 


\section{ACKNOWLEDGEMENT}

The authors would like to thank the referee for a number of useful comments.

\section{REFERENCES}

1. A. Ancona, First eigenvalues and comparison of Green's functions for elliptic operators on manifolds or domains, J. Anal. Math. 72 (1997), 45-92. MR1482989 (98i:58212)

2. F. V. Atkinson, On second-order non-linear oscillations, Pacific J. Math. 5 (1955), 643-647. MR0072316 (17:264e)

3. C. BANDlE AND H. A. Levine, On the existence and non-existence of global solutions of reaction-diffusion equations in sectorial domains, Trans. Amer. Math. Soc. 316 (1989), 595622. MR937878 (90c:35118)

4. H. Berestycki, I. CAPUZZO-Dolcetta And L. Nirenberg, Superlinear indefinite elliptic problems and nonlinear Liouville theorems, Topol. Methods Nonlinear Anal. 4 (1994), 5978. MR.1321809 (96d:35041)

5. H. Berestycki, L. Nirenberg and S. R.S. Varadhan, The principal eigenvalue and maximum principle for second-order elliptic operators in general domains, Comm. Pure Appl. Math. 47 (1994), no. 1, 47-92. MR.1258192 (95h:35053)

6. M.-F. BidAut-VÉron, Local and global behavior of solutions of quasilinear equations of Emden-Fowler type, Arch. Rational Mech. Anal. 107 (1989), 293-324. MR1004713 (90f:35066)

7. M.-F. Bidaut-Véron And S. Pohozaev, Non-Existence results and estimates for some nonlinear elliptic problems, J. Anal. Math. 84 (2001), 1-49. MR1849197(2002f:35085)

8. I. Birindelli AND E. MitidieRI, Liouville theorems for elliptic inequalities and applications, Proc. Roy. Soc. Edinburgh Sect. A 128 (1998), 1217-1247. MR.1664101 (99m:35277)

9. B. GIDAS AND J.SPRUCK, Global and local behavior of positive solutions of nonlinear elliptic equations, Comm. Pure Appl. Math. 34 (1981), 525-598. MR615628 (83f:35045)

10. D. Gilbarg and N.S. Trudinger, Elliptic Partial Differential Equations of Second-Order. Second edition, Springer, Berlin, 1983. MR737190 (86c:35035)

11. L. V. Kantorovich and G. P. Akilov, Functional Analysis. Second edition. Pergamon Press, Oxford-Elmsford, N.Y., 1982. MR664597(83h:46002)

12. V. A. Kondratiev AND E. M. LANDIS, Qualitative properties of the solutions of a second-order nonlinear equation, Mat. Sb. (N.S.) 135(177) (1988), 346-360; translation in Math. USSR-Sb. 63 (1989), 337-350. MR937645 (89j:35054)

13. V. KondratieV, V. LISKEVICH AND Z. SOBOL, second-order semilinear elliptic inequalities in exterior domains, J. Differential Equations 187 (2003), 429-455. MR1949449(2005b:35082)

14. V. Kondratiev, V. Liskevich, Z. Sobol and A. Us, Estimates of heat kernels for a class of second-order elliptic operators with applications to semi-linear inequalities in exterior domains, J. London Math. Soc. 69 (2004), 107-127. MR2025330 (2004j:35117)

15. V. Kondratiev, V. Liskevich and V. Moroz, Positive solutions to superlinear second-order divergence type elliptic equations in cone-like domains, Annales de l'Institut Henri Poincaré (C) Analyse Non Linéaire, 22 (2005), 25-43. MR2114410(2005i:35089)

16. V. Kondratiev, V. Liskevich, V. Moroz and Z. Sobol, A critical phenomenon for sublinear elliptic equations in cone-like domains, Bull. London Math. Soc. 37 (2005), 585-591. MR.2143738 (2006g:35067)

17. N. Meyers AND J.SERRIN, The exterior Dirichlet problem for second-order elliptic partial differential equations, J. Math. Mech. 9 (1960), 513-538. MR0117421(22:8200)

18. E. Mitidieri AND S. I. PohožAev, A priori estimates and the absence of solutions of nonlinear partial differential equations and inequalities (Russian), Tr. Mat. Inst. Steklova 234 (2001), 1-384. MR.1879326 (2005d:35004)

19. N. Nadirashvili, Nonuniqueness in the martingale problem and the Dirichlet problem for uniformly elliptic operators, Ann. Scuola Norm. Sup. Pisa Cl. Sci. (4) 24 (1997), 537-549. MR:1612401 (99b:35042)

20. Y. PINCHOVER, On the equivalence of Green functions of second-order elliptic equations in $R^{n}$, Differential Integral Equations 5 (1992), 481-493. MR 1157482 (93b:35035)

21. J. SERRIN AND H. ZOU, Cauchy-Liouville and universal boundedness theorems for quasilinear elliptic equations and inequalities, Acta Math. 189 (2002), 79-142. MR1946918(2003j:35107) 
22. J.S. W. Wong, On the generalized Emden-Fowler equation, SIAM Rev. 17 (1975), 339-360. MR0367368 (51:3610)

23. QI S. ZHANG, An optimal parabolic estimate and its applications in prescribing scalar curvature on some open manifolds with Ricci $\geq 0$, Math. Ann. 316 (2000), 703-731. MR 1758450 (2001b:53040)

24. QI S. ZhANG, A Liouville type theorem for some critical semilinear elliptic equations on noncompact manifolds, Indiana Univ. Math. J. 50 (2001), 1915-1936. MR1889088(2002k:35103)

Department of Mathematics and Mechanics, Moscow State University, Moscow 119 899, Russia

E-mail address: kondrat@vnmok.math.msu.su

Department of Mathematics, Swansea University, Swansea SA2 8PP, United Kingdom

E-mail address: V.A.Liskevich@swansea.ac.uk

Department of Mathematics, Swansea University, Swansea SA2 8PP, United Kingdom

E-mail address: z.sobol@swansea.ac.uk 